Depth distribution of primary cutaneous melanomas diagnosed in Nottingham health district residents in 1984-7. Results are numbers (and percentages)

\begin{tabular}{ccccc}
\hline & \multicolumn{3}{c}{ Breslow thickness $(\mathrm{mm})$} & $\begin{array}{c}\text { Annual } \\
\text { incidence } \\
\text { per } 100000 \\
\text { residents }\end{array}$ \\
\cline { 2 - 5 } Year & $<1.5$ & $1.5-3.49$ & $\geqslant 3.5$ & 5.4 \\
\hline 1984 & $14(52)$ & $8(30$ & $5(19)$ & 4.6 \\
1985 & $19(56)$ & $7(21)$ & $8(24)$ & 5.6 \\
1986 & $27(64)$ & $9(21)$ & $614)$ & 6.9 \\
1987 & $29(63)$ & $11(24)$ & $6(13)$ & $7 \cdot 5$ \\
\hline
\end{tabular}

preventive message the importance of early diagnosis of the disease must be emphasised. The value of local education campaigns must not, however, be taken for granted. Our experience highlights the need to provide resources to meet demand generated by a campaign. The Cancer Research Campaign, recognising this, funded two extra clinical assistant sessions a week. Despite this the extra referrals put considerable strain on staff, and without their good will a chaotic situation would have developed in the dermatology service. Districts interested in running local campaigns should first conduct an audit of the depth distribution of melanomas diagnosed in residents of their district so that the likely benefit to be gained from the inevitable increase in workload can be taken into account.

STEPHEN M WHITEHEAD

Department of Community Medicine, Southern Derbyshire Health Authority,

Derby DE1 2PH

Department of Dermatolog:

M A WROUGHTON

Nottingham NG7 2UH

\section{Idiopathic retroperitoneal fibrosis}

Mr Nigel Bullock gives a good summary of the clinical and pathological features of the uncommon but intriguing disease idiopathic retroperitoneal fibrosis (23 July, p 240). I think, however, that the subheading "Now known to be an allergic reaction to insoluble lipid leaking through arteries" is misleading and in some ways harmful and was not substantiated by any evidence quoted in the editorial. The two references cited, published in Atherosclerosis, do not provide proof of an allergic mechanism. They show the association of immunoglobulins with ceroid in atheromatous plaques, but the authors are more guarded in their interpretation than Mr Bullock suggests. There are other clinical and pathological features of the disease which suggest immunopathological mechanisms, although not particularly allergy to ceroid.

The misleading element of the subheading is the certainty with which it is proclaimed, and the harm is because of the widespread confusion in the application of the word "allergy" to clinical events.

From an immunological viewpoint the term should be applied only to interactions between antigens and either antibodies or sensitised lymphocytes that cause (through various effector mechanisms) physiological disturbances, perceived as symptoms or clinical signs, or both. Although the term "autoallergic" has been applied to instances when the antigens are derived from self, the term "autoimmunity" is more widely used and better understood in such circumstances, "allergy" being retained for symptomatic reactions to environmental non-self agents.

If indeed the leakage of ceroid provokes an immune response (and the evidence for this is not overwhelming) then surely this is an autoimmune reaction. There is also a big gap between showing immune responses to autoantigens and knowing that these cause the pathological manifestations of the disease in which they are found.

The loose use of "allergy" in this context is to be regretted, as well as the certainty with which the view is propounded, as an editorial in the $B M \mathcal{F}$.

R A THOMPSON

Regional Department of Immunology

East Birmingham Hospital,

Birmingham B9 5ST approach.

se of steroids would surely be the most logical

P M HIGGINS

G M ABER

P F NAISH DAVID BENNETT-JONES

North Staffordshire Health Authority

Central Outpatients Department,

Stoke on Trent,

AUTHORS' REPLY,-Dr Thompson is quite correct in suggesting that the presence of an immune response to autoantigens does not necessarily imply that clinicians know that this causes the recognised pathological manifestations of chronic periaortitis.

My intention was simply to suggest that there is good evidence that this may be the case but that it remains to be proved by clinicians and immunologists.

The subheading whose terminology he finds misleading was not part of my original manuscript but was a later editorial addition to summarise the essence of the article in a single phrase. As such, I agree that it does somewhat overstate the case. The point remains that the evidence in favour of an autoimmune aetiology is strong but, inevitably, not conclusive.

Addenbrooke's Hospital

NIGEL BULLOCK

Cambridge CB2 2QQ

We disagree strongly with $\mathrm{Mr}$ Nigel Bullock's statement in his editorial on idiopathic retroperitoneal fibrosis that "Definitive treatment necessitates laparotomy to free the ureters from the fibrotic plaque ... and wrapping of the ureters in omentum . . . to prevent recurrence." If "definitive" means anything it means that the episode is then concluded, whereas as he goes on to state-and as nearly all the authors quoted by him agree-“disease activity may persist or recur many years later.'

Mr Bullock accepts that many patients will respond to treatment with steroids so why reserve this form of treatment for those considered to be unfit for operation or for those who relapse after surgical treatment? All authors agree that operations on patients with retroperitoneal fibrosis carry risks, and in many cases it is difficult to see just what has been achieved by subjecting the patient to that risk.

Our recently published paper describes a series of 17 patients managed successfully without (in the main) surgical intervention. ${ }^{1}$ We would not claim to have restored function to all kidneys, but in patients with longstanding disease the renal damage will probably be irreversible whatever method of treatment is used, such patients being most at risk from operation. Those who present with acute disease will probably respond equally well to steroids and operation.

The main objection to non-operative management is the lingering doubt in the mind of the surgeon as to the correctness of the diagnosis, but this doubt can largely be dispelled by using modern diagnostic techniques, particularly computed tomography, and in most cases the response to steroid treatment soon establishes the diagnosis with certainty. It is noticeable that when the alternatives are explained to patients they find it easier to bear with equanimity the prospect of avoiding an operation than does the surgeon.

Although we acknowledge the possibility that retroperitoneal fibrosis may have an immunological basis, we believe that the evidence produced by $\mathrm{Mr}$ Bullock in support of this hypothesis carries no real conviction. The presence of lymphocytes and plasma cells in the perivascular lesions may well be a non-specific finding in any inflammatory mass, and the presence of immunoglobulins (IgG and IgM) could be due to non-specific "trapping." If, however, Mr Bullock really believes that this condition has an immunological basis the initial
1 Higgins PM, Bennett-Jones DN, Naish PF, Aber GM. Nonoperative management of retroperitoneal fibrosis. $\mathrm{Br} \mathcal{J}$ Surgen $1988 ; 75: 573-7$

\section{Amiodarone and thyroid immunity}

Dr A P Weetman and colleagues discussed thyroid autoimmunity in relation to amiodarone ( 2 July, $p$ 33). The drug could affect the thyroid directly because of its high iodine content or it may initiate an autoimmune process. The iodine content could be relevant to autoimmunity: in the study by Boukis et al in which patients in an iodine depleted area were given iodised oil thyroid disturbances with autoantibodies resulted. ${ }^{1}$ The drug could also because of its structural homology to iodothyronines interfere with control of the thyroid or with peripheral actions of thyroid hormones. The variable response to the drug may arise from differences in iodine state or immunogenetic make up.

There are several points arising from the study by Dr Weetman and others. All patients included were probably iodine replete. The relative incidence of antibody positive and negative thyrotoxicosis varies throughout the United Kingdom according to iodine state. ${ }^{2}$ It may be impossible to generalise about the pathogenesis of thyroid disturbances for the entire country from a study in the south east of England, which is iodine replete. The indications for treatment with amiodarone were not discussed but may be relevant. Thyroiditis with autoantibodies is commoner in ischaemic heart disease, irrespective of iodine state. ${ }^{3}$ This could account for the alarmingly high incidence of autoantibodies before amiodarone treatment, although the assay used was extremely sensitive. Using a similar assay we have found thyroid antibodies in up to $10 \%$ of normal subjects. In contrast to Dr Weetman and others Monteiro et al found a clear relation between thyroid autoantibodies and thyroid state after amiodarone. ${ }^{4}$ This study included a defined group of patients in the weeks after myocardial infarction. The time at which patients are studied matters. During short term administration of amiodarone there is release of thyroxine from the gland which is clearly not due to autoimmunity. ${ }^{5}$ The study of Monteiro et al was short term whereas that of Dr Weetman and others was largely long term.

Hyperthyroidism is less common than hypothyroidism after amiodarone treatment and is usually transient. It may result from release of hormone during silent thyroiditis. This could explain the variable relation among thyroid stimulating hormone concentration, autoantibodies, and thyroid state which is documented. It is not surprising, therefore, that stimulating immunoglobulins were not detected in this study. We have not, in any case, found the assay using the rat thyroid cell line FRTL5 to be particularly sensitive except in clear cut cases of Graves' disease. Furthermore, Dr Weetman and others did not state whether purified immunoglobulins were used and this will affect the sensitivity. Stimulatory immunoglobulins have been found in patients with established hyperthyroidism after amiodarone treatment by Omri-Delangen et al using an assay based on human thyroid cells. ${ }^{6}$ The efficacy of amiodarone coupled with its low incidence of serious side effects means that its use will continue 